\title{
Caracterização físico-química e sensorial de néctares de uva tradicionais e light
}

\author{
Marília Silveira SANTANA ${ }^{1}$ \\ Flávia DELLA LUCIA ${ }^{2}$ \\ Eric Batista FERREIRA ${ }^{3}$ \\ Marisa de Oliveira LOPES ${ }^{4}$
}

\begin{abstract}
${ }^{1}$ Nutricionista, UNIFAL-MG, marilia_silveira_123@yahoo.com.br; ${ }^{2}$ Professora Assistente, Laboratório de Técnica Dietética, Faculdade de Nutrição, UNIFAL-MG; flavia@unifalmg.edu.br;

${ }^{3}$ Professor Adjunto; Instituto de Ciências Exatas, UNIFAL-MG; eric.ferreira@unifal-mg.edu.br; ${ }^{4}$ Farmacêutica, Laboratório de Técnica Dietética, Faculdade de Nutrição, UNIFAL-MG; marisa.lopes@unifalmg.edu.br
\end{abstract}

Recebido em: 04/10/2012 - Aprovado em: 19/12/2012 - Disponibilizado em: 30/12/2012

\begin{abstract}
RESUMO
Este trabalho teve como objetivo avaliar a qualidade físico-química e sensorial de néctares de uva de marcas comerciais, nas versões tradicionais e light. Foram utilizadas quatro amostras, duas amostras foram adoçadas com sacarose, uma com ciclamato de sódio e sacarina sódica e uma com sucralose e acessulfame K. Procedeu-se às seguintes análises físico-químicas: coloração, sólidos solúveis totais (SST), acidez titulável, pH, relação SST/AT. Utilizou-se a escala hedônica (9 pontos) para avaliar a aceitabilidade sendo os dados analisados por ANAVA/ ScottKnott (5\% de probabilidade) e mapa de preferência interno. Constatou-se que a amostra B tradicional foi a que obteve maior média de aceitação e diferiu das demais amostras ( $\mathrm{p} \leq 0.05)$. A amostra $\mathrm{B}$ tradicional obteve maior índice de SST. As demais amostras não atendem a atual legislação, sendo que para os produtos light não há valores recomendados para parâmetros físico-químicos. Em relação à \% AT, a amostra B tradicional apresentou a maior acidez e a versão light, a menor. Ao avaliar a relação SST/AT as amostras tradicionais se encontram dentro da faixa de recomendação. Em relação ao pH todas as amostras estão dentro dos padrões preconizados. Em relação à coloração a amostra B tradicional, diferiu das demais em relação $L^{*}, a^{*}, b^{*}$, sendo que apresentou a menor luminosidade, que é a mais aceita. Ao se analisar os valores $\mathrm{a}^{*} \mathrm{e} \mathrm{b}^{*}$ todas as amostras tenderam às cores vermelho e azul conferindo cor arroxeada.

Palavras-chave: Aceitabilidade. Qualidade. Néctar de uva. Mapa de Preferência. Escala hedônica.
\end{abstract}

\begin{abstract}
This study aimed to evaluate the physicochemical and sensory grape nectar trademarks, traditional and light versions. Four samples were used. The two of them sweetened with sucrose, one sweetened with a sodium cyclamate and sodium saccharin and one with sucralose and acesulfame K. The following physical-chemical analyses were carried out: color, total dissolved solids, titratable acidity, $\mathrm{pH}, \mathrm{TSS} / \mathrm{TA}$ relationship. The Hedonic scale ( 9 points) was used to evaluate the acceptance with the data analyzed by ANOVA / Scott-Knott test ( 5\% probability) and internal preference mapping. It was found that the B traditional sample was the one that had the highest acceptance average and differed from the other samples $(\mathrm{p} \leq 0.05)$.The B traditional sample had the highest SST rate. The remaining samples do not meet the current legislation. Regarding the AT \% B traditional sample has the highest acidity and the light version to lower acidity. When evaluating the TSS / TA samples lie within the traditional range of recommendation. Regarding the $\mathrm{pH}$ all samples meet the recommended standards. Regarding to coloring the B traditional sample, differed from the others in $\mathrm{L}$ $*, a *, b *$, and showed lowest luminosity, which is the most accepted. When analyzing the $\mathrm{a} *$ and $\mathrm{b} *$ values all samples tended to the red and blue colors bestowing purple color.
\end{abstract}

Keywords: Acceptability. Quality. Grape nectar. Preference map. Hedonic scale.

\section{INTRODUÇÃO}

O néctar de frutas é a bebida não potável da parte comestível da fruta e açúcares; ou de extratos vegetais e açúcares. Admite ainda a adição de ácidos e é destinada ao consumo direto (BRASIL, 1997). 
O suco de uva é uma importante fonte de antioxidantes naturais devido ao seu elevado teor de substâncias fenólicas. As antocianinas são as principais responsáveis pela cor do suco, as quais podem sofrer degradações e transformações durante o processamento e armazenamento do produto (GURAK et al., 2008). O suco de uva apresenta efeitos como manutenção da função endotelial, diminuição da agregação plaquetária, aumento da capacidade antioxidante e proteção contra oxidação do LDL colesterol (PONTES et al., 2010).

A busca pela qualidade de vida se estende aos cuidados com a alimentação, caracterizada por uma crescente demanda por produtos saudáveis e com características nutricionais e sensoriais próximas dos alimentos in natura (ENDO et al., 2009).

As informações obtidas através de avaliações sensoriais podem ser utilizadas por empresas como suporte técnico para pesquisa, industrialização, marketing e controle de qualidade dos itens produzidos, e para apoiar decisões administrativas. $\mathrm{Na}$ perspectiva do consumidor, a avaliação sensorial em produtos industriais assegura que os mesmos cheguem ao mercado com as características desejadas (ROSSINI; ANZANELLO; FOGLIATTO, 2009). A indústria de alimentos está atenta ao mercado consumidor e tem desenvolvido produtos e tecnologias para a produção de alimentos com baixas calorias, mediante substituição de açúcar por edulcorantes e/ou redução no seu teor de gordura (CÂNDIDO; CAMPOS, 1996).

\section{MATERIAIS E MÉTODOS}

\section{Caracterização das amostras}

Foram utilizadas 2 marcas comerciais de néctar de uva em embalagens cartonadas, nas versões tradicional e light, sendo duas amostras adoçadas com sacarose (A e B tradicional), uma com ciclamato de sódio e sacarina sódica (A Light) e uma com sucralose e acessulfame K (B Light). Adquiriram-se 4 embalagens de cada uma das amostras, sendo homogeneizadas no momento das análises. As informações nutricionais contidas nos rótulos dos produtos são mostradas na Tabela 1.

Tabela 1 - Informações nutricionais contidas nos rótulos das amostras de néctares de uva

\begin{tabular}{|c|c|c|c|c|}
\hline \multirow{2}{*}{$\begin{array}{c}\text { Informações } \\
\text { nutricionais } \\
(200 \mathrm{~mL})\end{array}$} & \multicolumn{4}{|c|}{ Marcas } \\
\hline & BT & $\begin{array}{l}\mathbf{B} \\
\mathbf{L}\end{array}$ & AT & $\mathbf{A L}$ \\
\hline Valor Calórico (kcal) & 120 & $\begin{array}{c}39 \\
4\end{array}$ & 100 & 40 \\
\hline Carboidratos (g) & 29 & $\begin{array}{c}9,5 \\
5\end{array}$ & 25 & 10 \\
\hline Sódio (mg) & 7,6 & $\begin{array}{c}7,1 \\
6\end{array}$ & 4,8 & 18 \\
\hline Vitamina C (mg) & $\mathrm{nc}^{*}$ & $\begin{array}{c}\mathrm{nc} \\
*\end{array}$ & 9 & 26 \\
\hline
\end{tabular}

\section{Análises Físico-químicas}

Realizaram-se as seguintes análises de acordo com o estabelecido na Legislação Brasileira: $\mathrm{pH}$, acidez total titulável, coloração e sólidos solúveis totais. Os procedimentos 
foram realizados em 3 repetições em delineamento inteiramente casualizado.

A avaliação da cor foi realizada por meio de colorímetro (Konica Minolta® CR-10/CIE L* $\left.a^{*} b^{*}\right)$, com leituras realizadas em 4 pontos distintos da amostra dispostas em placa de petri. A coordenada $\mathrm{L}^{*}$ representa o quanto mais claro ou mais escuro é a amostra, com valores variando de 0 (totalmente preta) a 100 (totalmente branca). Já a coordenada $a^{*}$ pode assumir valores de -80 a +100 , em que os extremos correspondem ao verde e ao vermelho, respectivamente. Por fim, a coordenada $b^{*}$, com a intensidade de azul ao amarelo, pode variar de -50 (totalmente azul) a +70 (totalmente amarelo). O Teor de Sólidos Solúveis (SS) foram determinados por refratometria (ATAGO®), expressos em ${ }^{\circ}$ Brix, conforme a AOAC (1998).

A acidez titulável foi determinada segundo INSTITUTO ADOLFO LUTZ (1985), com resultados expressos em porcentagem de ácido tartático. A determinação do $\mathrm{pH}$ foi realizada por pHmetro (TECNOPON ( $\mathrm{M}$ PA-210) conforme AOAC (1992). Para o cálculo da relação SST/AT foi realizada a divisão do teor de sólidos solúveis totais pela acidez titulável.

\section{Teste de Aceitação}

Os indivíduos foram recrutados através de questionário onde se excluiu aqueles que não gostavam do produto. Os testes sensoriais foram realizados no Laboratório de Técnica Dietética da Faculdade de Nutrição (UNIFAL-
MG), em cabines individuais por 100 consumidores de néctar de uva. Os consumidores avaliaram a aceitação global através de escala hedônica de 9 pontos (9 gostei extremamente; 1 - desgostei extremamente), avaliando -se também o que mais gostaram e desgostaram em cada amostra.

As amostras foram servidas em uma única sessão em copos descartáveis de $50 \mathrm{ml}$ codificados com número de três dígitos aleatórios, ordenados de forma casualizada. Foi fornecido um copo d'água para enxaguar a boca entre as amostras.

\section{Análises Estatísticas}

O experimento foi conduzido em delineamento em blocos completos casualizados e análise dos resultados por análise de variância (ANAVA), comparação de médias pelo Teste de Scott-Knott (ambos a 5\% de significância) e Mapa de Preferência Interno. Para todas as análises foi utilizado o $\mathrm{R}$ versão 2.13.2 (R. DEVELOPMENT CORE TEAM, 2011), por meio dos pacotes ExpDes (CAVALCANTI, 2010) e SensoMineR (HUSSON; LÊ; CADORET, 2011).

\section{RESULTADOS E DISCUSSÃO}

Nos resultados da análise sensorial através da anáise de variância, realizado pelo Teste de Scott-Knott para a comparação das médias, constatou-se que a marca B Tradicional foi a 
que obteve a maior média de aceitação $(6,9$ entre os termos hedônicos "gostei ligeiramente" e "gostei moderadamente") e diferiu das demais amostras. As demais amostras não diferiram entre si ( $\mathrm{p} \geq 0.05)$. Para as amostras A Light e A Tradicional foram encontradas as médias 6,5 e 6,38, respectivamente. A marca B Light foi a amostra que obteve a menor média aceitação $(6,22)$, onde todas amostras apresentaram uma boa aceitação.

Sucos adoçados parecem ter a preferência do consumidor, conforme demonstrado por Matsuura et al. (2004) no desenvolvimento de um néctar à base de polpa de mamão e suco de maracujá. Branco et al. (2007) verificaram que o teor de sacarose (5 ou 10\%) não influenciou a aceitação de um blend de suco de laranja e cenoura.

O Mapa de preferência interno (Figura 1) foi construído de forma a considerar as preferências individuais de cada provador. Assim, as 100 respostas individuais dos provadores, com relação a cada amostra de néctar de uva avaliada, geraram um espaço sensorial multidimensional representado por dimensões que explicam a variação total das respostas. Os vetores caracterizam a aceitação individual de cada provador. $\mathrm{O}$ primeiro componente explicou aproximadamente $39,77 \%$ da variância total, e o segundo, $36,94 \%$, perfazendo $76,71 \%$ de explicação da variância total dos dados de aceitação.

Figura 1. Círculo de correlação e gráfico de dispersão das amostras de néctar de uva analisadas.
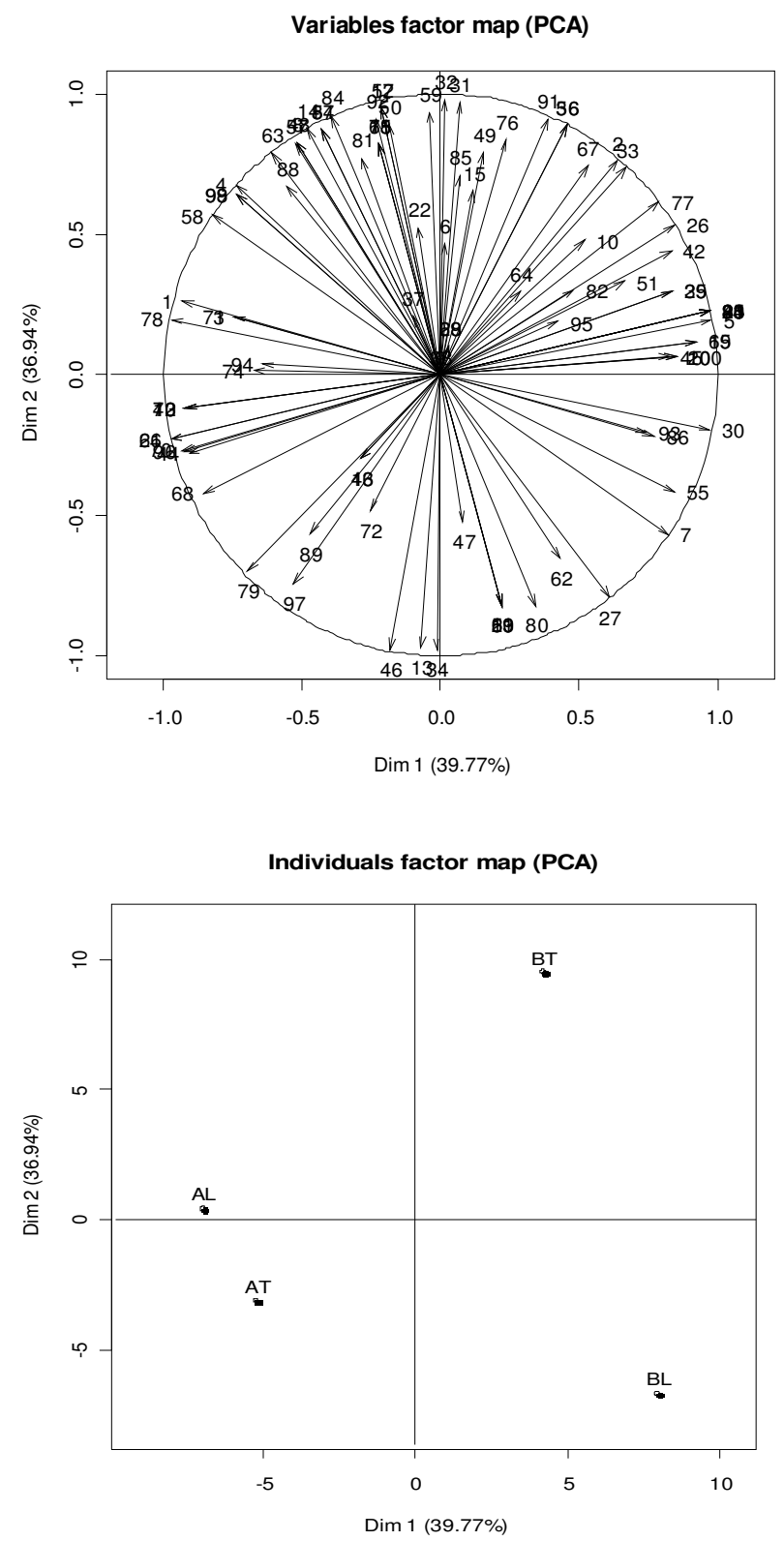

Legenda: AL (A Light), BL (B Light), AT (A tradicional) e BT (B tradicional)

Houve uma aglomeração ligeiramente maior no $1^{\circ}$ quadrante. Este resultado vai de encontro ao apresentado na análise de variância, onde também a amostra B Tradicional foi a mais aceita e se diferenciou das demais. Observa-se a formação de um segundo grupo (amostras A Light e A Tradicional; segundo e terceiro quadrantes, respectivamente) e um terceiro grupo formado 
pela amostra B Light (4 ${ }^{\circ}$ quadrante). Estes resultados também confirmam o encontrado na ANAVA, mostrando uma menor aceitação para as amostras B Light e A tradicional do que a B Tradicional. Entretanto, a amostra A tradicional mostra semelhança em relação a sua versão light.

Os dados referentes à frequência do que os consumidores consideraram mais gostar ou desgostar nas amostras são expostos nas Tabelas 2 e 3.

Tabela 2 - Frequência de aparecimento dos termos mais citados como "O que mais gostou" nas amostras de néctar de uva analisados.

\begin{tabular}{|c|c|c|}
\hline Marcas & Termos citados & $\begin{array}{c}\text { Frequência de } \\
\text { aparecimento } \\
(\%)\end{array}$ \\
\hline \multirow[t]{4}{*}{ B Light } & Adocicado & 35 \\
\hline & Sabor & 20 \\
\hline & Aroma & 10 \\
\hline & Cor & 7,5 \\
\hline \multirow[t]{4}{*}{ B Tradicional } & Adocicado & 26 \\
\hline & Sabor & 26 \\
\hline & Mais concentrado & 14 \\
\hline & Cor & 11 \\
\hline \multirow[t]{4}{*}{ A Light } & Sabor & 25 \\
\hline & Cor & 10 \\
\hline & Adocicado & 14,5 \\
\hline & Suave & 8 \\
\hline \multirow[t]{4}{*}{ A Tradicional } & Sabor & 27 \\
\hline & Adocicado & 15 \\
\hline & Gosto forte & 11 \\
\hline & Cor & 6 \\
\hline
\end{tabular}

Observa-se que em todas as amostras comparadas, os termos mais citados em relação à aceitação foram adocicado, sabor $e$ cor.
Tabela 3 - Frequência de aparecimento dos termos mais citados como "O que menos gostou" nas amostras de néctar de uva analisados.

\begin{tabular}{|c|c|c|}
\hline Marcas & Termo & $\begin{array}{c}\text { Frequência de } \\
\text { aparecimento }(\%)\end{array}$ \\
\hline & Adocicado & 20 \\
\hline \multicolumn{3}{|l|}{ B Light } \\
\hline & Sem açúcar & 10 \\
\hline & Ralo & 13,5 \\
\hline & Gosto fraco & 13,5 \\
\hline & Adocicado & 12 \\
\hline \multicolumn{3}{|l|}{ B Tradicional } \\
\hline & Ácido & 10 \\
\hline & Sabor & 19 \\
\hline & Gosto forte & 12 \\
\hline & Sem açúcar & 10 \\
\hline \multicolumn{3}{|l|}{ A Light } \\
\hline & Ácido & 12 \\
\hline & Sabor & 10 \\
\hline & Ralo & 10 \\
\hline & Ácido & 14 \\
\hline \multicolumn{3}{|l|}{ A Tradicional } \\
\hline & Amargo & 7 \\
\hline & Sabor & 7 \\
\hline & Gosto fraco & 7 \\
\hline
\end{tabular}

Em relação aos dados sobre os motivos da não aceitação, ao se comparar as versões Light e Tradicional da marca B predominou-se o termo adocicado. Já quando compara-se as mesmas versões para a marca A predominaram-se os termos sabor e ácido. Para as amostras A Light e B Light, quando comparadas verifica-se os termos ralo e sem açúcar e para as amostras B Tradicional e A Tradicional, observa-se os termos sabor $e$ ácido.

$\mathrm{Na}$ Tabela 4, observa-se que apenas a amostra A, tanto a tradicional quanto a Light, utiliza a polpa de fruta como espessante natural, o que não ocorre com a marca $\mathrm{B}$, que utiliza o espessante goma xantana. Todas as amostras apresentam acidulante ácido cítrico, 
que é utilizado como ácido forte, aromatizante e antioxidante em sucos de frutas, responsável pela acidez de certas frutas cítricas (FIORUCCI et al., 2002).

Em relação ao Néctar de uva, era esperado que as amostras A e B tradicionais, por não conterem edulcorantes apresentassem maior aceitabilidade em relação às amostras light. Verificou-se que a amostra B tradicional obteve maior aceitabilidade. A amostra A light obteve maior aceitação do que a amostra $\mathrm{A}$ tradicional. Os resultados demonstraram o inverso do esperado, provavelmente devido a utilização de ciclamato de sódio/sacarina sódica que apresenta grande semelhança ao sabor conferido pela sacarose (BIACCHI, 2006).

As amostras A, tanto Tradicional quanto Light utilizam ácido ascórbico cuja presença parece não influenciar na aceitabilidade, pois não se obteve aceitabilidade inferior na amostra A.

Tabela 4 - Ingredientes informados nos rótulos de néctares de uva comerciais analisados.

\begin{tabular}{|c|c|c|c|c|}
\hline & \multicolumn{4}{|c|}{ Ingredientes } \\
\hline Marca & Acidulante & Edulcorante & Espessante & Antioxidante \\
\hline $\mathrm{AT}$ & $\begin{array}{l}\text { Ácido } \\
\text { cítrico }\end{array}$ & - & polpa & $\begin{array}{c}\text { Ácido } \\
\text { Ascórbico }\end{array}$ \\
\hline $\mathrm{AL}$ & $\begin{array}{l}\text { Ácido } \\
\text { cítrico }\end{array}$ & $\begin{array}{l}\text { Ciclamato de } \\
\text { sódio e } \\
\text { Sacarina } \\
\text { sódica }\end{array}$ & polpa & $\begin{array}{c}\text { Ácido } \\
\text { Ascórbico }\end{array}$ \\
\hline BT & $\begin{array}{l}\text { Ácido } \\
\text { cítrico }\end{array}$ & - & $\begin{array}{c}\text { Goma } \\
\text { Xantana }\end{array}$ & - \\
\hline$\overline{B L}$ & $\begin{array}{l}\text { Ácido } \\
\text { cítrico }\end{array}$ & $\begin{array}{c}\text { Sucralose e } \\
\text { Acessulfame } \\
\text { K }\end{array}$ & $\begin{array}{c}\text { Goma } \\
\text { Xantana }\end{array}$ & - \\
\hline
\end{tabular}

Legenda: $\mathrm{S}=\mathrm{Sim} ; \mathrm{N}=$ Não $; \mathrm{BT}=\mathrm{B}$ Tradicional; $\mathrm{BL}=\mathrm{B}$

Light; AT $=$ A Tradicional; $\mathrm{AL}=\mathrm{A}$ Light
Na amostra A Light, foi feita a associação de ciclamato de sódio e sacarina sódica e na amostra B Light foi feita a associação de sucralose e acessulfame de potássio. As amostras A, tanto a tradicional quanto a Light, utilizam o ácido ascórbico que tem o poder antioxidante. Já as amostras B, não utilizam esse ácido. Em relação aos edulcorantes utilizados na amostra A Light foi feito a associação de ciclamato de sódio e sacarina sódica. $\mathrm{Na}$ amostra $\mathrm{B}$ Light foi feito a associação de sucralose e acessulfame de potássio. O grande problema da associação de ciclamato de sódio e sacarina sódica, é que além de ambos serem artificiais, possuem grande quantidade de sódio, que traz prejuízos aos hipertensos (COELHO, 2010). Apesar da sacarina apresentar gosto residual amargo e metálico em altas concentrações, esse efeito indesejável pode ser minimizado associando-a com outro edulcorante como, por exemplo, o ciclamato. Essa associação é muito empregada, pois o ciclamato mascara as notas de sabor residual amargo da sacarina e esta, por sua vez, potencializa o baixo poder edulcorante do ciclamato (RÖDEL; GUIDOLIN, 2006).

Os dados referentes aos parâmetros físicoquímicos são expostos na Tabela 5.

Em relação aos teores de SST, evidencia-se que este parâmetro reflete, dentre outros fatores, os níveis de açúcares presentes nos néctares de uva avaliados. Observa-se que a marca B Tradicional apresentou maior índice de sólidos solúveis totais, perfazendo um total 
de $14,56^{\circ}$ Brix, sendo que para a marca $\mathrm{A}$ Tradicional foram encontrados níveis menores de $\operatorname{SST}\left(12,97^{\circ}\right.$ Brix). A amostra A Light mostrou teores de $6,73^{\circ}$ Brix e para B Light teores de $4,63^{\circ}$ Brix. Todas as amostras diferiram entre si ao nível de $5 \%$ de probabilidade pelo Teste de Scott-Knott.

Tabela 5 - Parâmetros físico-químicos das diferentes marcas comerciais de néctar de uva.

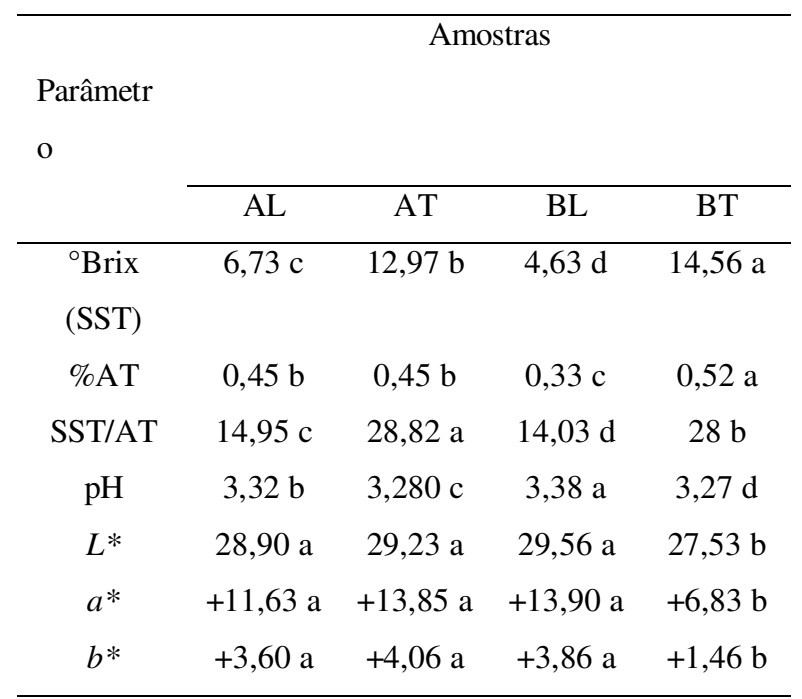

Legenda: Médias seguidas de mesma letra na linha não diferem entre si ao nível de 5\% de probabilidade pelo teste de Scott-knott. AT: A Tradional; AL: A Light; BT: B Tradicional; BL: B Light ; SST: Sólidos solúveis totais; AT: acidez titulável; $* \mathrm{~L}=$ Luminosidade $(0=$ preto, $100=$ branco $)$; *a (-80: verde; +100: vermelho); *b (-50: azul; +70: amarelo); \%AT: \% ácido tartárico.

Com base na exigência legal para os teores de sólidos solúveis totais, apenas a marca B Tradicional apresenta-se adequada na avaliação deste parâmetro. As demais amostras apresentam valores inferiores a $14^{\circ}$ Brix, não atendendo aos padrões preconizados. Justificase a diferença entre os valores encontrados nas versões light e tradicionais pelo fato de que no light não há adição de sacarose e sim de edulcorantes em quantidades muito menores comparativamente. A amostra B Tradicional também apresentou a maior média de aceitação, o que pode estar correlacionado ao seu maior teor de sólidos solúveis.

Gurak (2008) avaliou os teores de sólidos solúveis nos néctares de uva e encontrou valores entre 11,0 a $14,5^{\circ}$ Brix para as amostras sem redução do valor calórico e valores entre 4,5 a 5,0 $0^{\circ}$ Brix para aquelas com redução por meio de substituição por variados tipos de edulcorantes (aspartame, ciclamato de potássio, sacarina e acessulfame de potássio). Estes resultados vão de encontro com os obtidos no presente estudo, visto que a variação foi de 12,97 a 14,56 e 4,63 a $6,73^{\circ}$ Brix, para amostras tradicionais e light, respectivamente.

No que concerne aos níveis de acidez titulável de sucos de uva, a Legislação Brasileira estabelece um mínimo de 0,41g de Ácido Tartárico em $100 \mathrm{ml}$ de suco ou $0,41 \%$ (BRASIL, 2004). Deste modo, apenas a marca B Light encontra-se com valor inferior ao mínimo estabelecido $(0,33 \%)$. As marcas A Light e A Tradicional não apresentaram diferença significativa; e a marca B Tradicional apresentou valor superior as demais amostras de $0,52 \%$ e diferiu significativamente das demais amostras.

Girard (1998) relata que a presença de níveis de acidez elevados em sucos de uva é justificada pelo conteúdo de ácidos orgânicos das uvas, com predomínio dos ácidos tartárico, málico e cítrico, podendo a distribuição destes compostos variam de acordo com a cultivar da uva. Contudo, ressalta-se que a acidez 
assegurada por estes ácidos, em equilíbrio com os açúcares da uva, são os responsáveis pelo sabor característico do suco. A amostra B tradicional foi a mais bem aceita e possui uma maior acidez, podendo esta informação sugerir uma importante característica de direcionamento de aceitabilidade.

Outro atributo avaliado nos sucos foi a relação SST/AT, considerada um indicativo de qualidade do suco de uva, uma vez que traça um parâmetro entre quantidades de açúcares e ácidos presentes na fruta e assim define as características de sabor do suco. Neste contexto, a legislação recomenda que os valores de relação SST/AT estejam entre 15 e 45 (BRASIL, 1998), sendo que valores situados fora desta faixa podem descaracterizar o sabor do suco (NATIVIDADE, 2010). Todas as amostras diferiram entre si para a relação SST/AT ao nível de $5 \%$ de probabilidade pelo Teste de Scott-knott. As amostras A Tradicional e B Tradicional apresentam maiores médias $(28,82$ e 28,0; respectivamente), onde apresentam valores dentro da faixa de recomendação, devido à quantidade de sólidos solúveis presentes. Já as amostras B Light e A Light apresentaram valores menores (14,03 e 14,95; respectivamente), onde apresentam valores abaixo da faixa de recomendação, porém não há valores recomendados para os parâmetros físico-químicos para produtos light.

$\begin{array}{ccc}\text { Provavelmente } & \text { apresentaram valores } \\ \text { menores devido a quantidade de } & \text { sólidos }\end{array}$ solúveis presentes serem menores nas versões light.

Em relação ao $\mathrm{pH}$, todas as amostras diferiram entre si ao nível de $5 \%$ de probabilidade pelo Teste de Scott-Knott. Observa-se que houve diferença significativa, sendo que a marca B Tradicional apresentou características mais ácidas em relação as demais, com pH de 3,27. A marca B Light apresentou valores de $\mathrm{pH} 3,38$, sendo esta a maior média. Todas as amostras apresentaram valores superiores a pH 2,9 e atendem aos padrões preconizados .

Em relação à coloração, a marca $\mathrm{B}$ Tradicional diferiu das demais amostras, tanto para $L^{*}, a^{*}$ e $b^{*}$, e também foi a amostra que obteve maior aceitação. As demais amostras não diferiram entre $\mathrm{si}(\mathrm{p} \geq 0.05)$.

Em relação ao valor de $a^{*}$, a amostra $B$ Tradicional obteve menor valor $(+6,83)$ que as demais amostras e também foi a amostra de maior aceitação. A amostra B Light obteve maior valor $(+13,90)$, sendo a amostra de menor aceitação. Todas as amostras para o valor de $\mathrm{a}^{*}$ tendem à coloração vermelha.

Os valores de $b^{*}$ demonstram que a amostra A Tradicional obteve maior valor $(+4,06)$, que as demais amostras, sendo que a amostra B Tradicional obteve menor valor $(+1,46)$, e apresenta maior aceitação. Todas as amostras para o valor de $b$ tendem à coloração azul.

Os valores $\mathrm{a}^{*} \mathrm{e} \mathrm{b}^{*}$ das amostras de néctar de uva mostram uma tendência às cores vermelho e azul (valores próximos de 0 tendendo ao azul), conferindo uma tonalidade 
arroxeada, onde a mistura das cores azul e vermelho resultam na coloração magenta. A marca B Tradicional apresentou menor luminosidade que as demais, o que pode ter contribuído para sua melhor aceitação no teste sensorial. $O$ inverso pode ser observado na amostra B Light que apresentou maior luminosidade que as demais (Tabela 5).

\section{CONCLUSÃO}

Analisando as amostras de Néctares de uva, nas versões Tradicionais e Light verificou-se que a amostra $\mathrm{B}$ tradicional foi a mais bem aceita e mostrou maior acidez e teor de SST, além de uma boa relação SST/AT e um menor valor de $\mathrm{L}^{*}$, sugerindo importantes características de direcionamento de aceitabilidade

Em relação ao $\mathrm{pH}$, todas se encontram dentro dos valores estabelecidos pela legislação. Para o SST ( ${ }^{\circ}$ Brix) apenas a amostra B tradicional atende à legislação, as demais apresentam valores inferiores ao recomendado. As amostras A e B tradicionais se encontram dentro dos valores estabelecidos para SST/AT na legislação, as amostras A e B light apresentaram-se fora destes padrões, pois então não há valores estabelecidos pela legislação para este tipo de produto light, sendo analisadas de acordo com a legislação para o produto tradicional.

\section{REFERÊNCIAS}

ASSOCIATION OF OFFICIAL AGRICULTURAL CHEMISTS - AOAC. Official methods of analysis of the Association of the Agricultural Chemists. 12 ed. Washington: A.O.A.C., 1992.

ASSOCIATION OF OFFICIAL ANALYTICAL CHEMISTS - AOAC. Official Methods of Analysis, 16 ed. Washington: A.O.A.C., 1998.

BIACCHI, S. M. Análise qualitativa e sensorial de edulcorantes em bolo caseiro. UNIFRA - Santa Maria, RS, 2006.

BRANCO, I. G., et al. Avaliação sensorial e estabilidade físico-quimica de um blend de laranja e cenoura. Revista de Ciência e Tecnologia de Alimentos, v. 27, n. 1, p. 7-12, 2007.

BRASIL. Ministério da Saúde. Agência Nacional de Vigilância Sanitária. Regulamenta a Lei $\mathrm{n}^{\circ} 8.918,14$ de julho de 1994, que dispõe sobre a padronização, a classificação, o registro, a inspeção, a produção e a fiscalização de bebidas. Decreto $n^{\circ} 2314$, de 04 de setembro de 1997. Diário Oficial da União, Brasília, 1997.

BRASIL. Ministério da Saúde. Portaria ${ }^{\circ}$ 544, de 16 de Novembro de 1998. Aprova o Regulamento Técnico para Fixação dos Padrões de Identidade e Qualidade para Refresco. Diário Oficial da República Federativa do Brasil, Brasília, 10 novembro 1998.

BRASIL. Ministério da Agricultura, Pecuária e Abastecimento. Secretaria de Defesa Agropecuária. Complementação dos Padrões de Identidade e Qualidade do Vinho e dos Derivados da Uva e do Vinho. Brasília (Portaria 55 de 30 de julho de 2004), p. 21, 2004.

CÂNDIDO, L. M. B.; CAMPOS, A. M. Alimentos para fins especiais: dietéticos. Livraria Varela, São Paulo, p. 60-61; 259-262; 115-122; 127-129, 1996. 
CAVALCANTI, P. P. Experimental Designs: um pacote $\mathrm{R}$ para análise de experimentos. 2010. p.90. Trabalho de Conclusão de Curso (Graduação em Biotecnologia). Universidade Federal de Alfenas, Alfenas, 2010.

COELHO, S. Liga da Saúde. Disponível em: $<$ http://ligadasaude.blogspot.com/2011/05/esc olhendo-melhor-seu-adocante.html $>$ Acesso em: 28 ago, 2010.

ENDO, E., et al. Caracterização do mercado consumidor de "água aromatizada": hábitos e motivações para o consumo. Ciência e Tecnologia de Alimentos, Campinas, abr-jun. 2009.

FIORUCCI, A. R., et al. Ácidos Orgânicos: dos Primórdios da Química Experimental à sua presença em nosso cotidiano. Química nova na escola, n.15, maio 2002.

GIRARD, B.; MAZZA, G. Produtos funcionales derivados de lãs uvas y de los cítricos. In: MAZZA, G.; ACRIBIA, S. A. Alimentos funcionales: aspectos bioquímicos e de processado. Zaragoza: Acribia, 1998. Cap. 5 , p. 141-182.

GURAK, P. D., et al. Avaliação de Parâmetros Físico-quimicos de sucos de uva integral, néctares de uva e néctares de uva light.

Revista de Ciências Exatas, RJ, v. 27, n. 1-2, 2008.

HUSSON, F.; LÊ, S.; CADORET, M.

SensoMineR: Sensory data analysis with R. R package version 1.14. 2011. Disponível em: http://CRAN.R-

project.org/package $=$ SensoMineR

INSTITUTO ADOLFO LUTZ. Normas Analíticas do Instituto Adolfo Lutz:

Métodos químicos e físicos para análises de alimentos. 3 ed., São Paulo: v.1, 1985.
MATSUURA, E. C. A. U., et al. Sensory acceptance of mixed néctar of papaya, passion fruit and acerola. Scientia Agricola, v. 61, n. 6, p. 604-608, 2004.

MINIM, V. P. R. Análise Sensorial. Estudos com Consumidores. Editora da UFV. 2006.

NATIVIDADE, M. M. P. Desenvolvimento, caracterização e aplicação tecnológicade farinhas elaboradas com resíduos da produção de suco de uva, Lavras: UFLA, 2010.

PONTES, P. R. B., et al. Atributos sensoriais e aceitação de sucos de uva comerciais. Ciência e Tecnologia Alimentos, Campinas, v. 30, $\mathrm{n}$. 2, p. 313-318, abr./jun. 2010 R. DEVELOPMENT CORE TEAM. R: A language and environment for statistical computing. R Foundation for Statistical Computing Vienna Austria. 2011. ISBN 3900051-07-0, URL http://www.R-project.org/

RÖDEL, N.; GUIDOLIN, F. R. Uso de edulcorantes. Serviço brasileiro de respostas técnicas. Disponível em: <http: //sbrt.ibict.br/ulpoad/sbrt3398.pdf> Acesso em: 2/10/2006.

ROSSINI, K; ANZANELLO, M. J; FOGLIATTO, F. S. Métodos para a seleção de atributos em avaliações sensoriais descritivas. Pesquisa Operacional na Gestão do Conhecimento. 2009.

SUPLICY, H. Adoçantes Artificiais. Revista da ABESO, ed. 49, Fevereiro, 2011.

Disponível:

<http://www.abeso.org.br/pagina/339/adocante s-artificiais.shtml> Acesso em: 23/10/2011.

\section{Agradecimentos}

À Universidade Federal de Alfenas, aos provadores e colaboradores. 